\title{
Ontogenia de los estróbilos, desarrollo de los esporangios y esporogénesis de Equisetum giganteum (Equisetaceae) en los Andes de Colombia
}

\author{
Edgar Javier Rincón Barón ${ }^{1,6}$, Helkin Giovani Forero Ballesteros ${ }^{2,6}$, Leidy Viviana Gélvez
}

Landazábal $^{2,6}$, Gerardo Andrés Torres ${ }^{3}$ \& Cristina Hilda Rolleri ${ }^{4,5}$

1. Grupo Semillero ecológico, Departamento de Biología, Calle 13 \# 100-00, Universidad del Valle, Sede Meléndez, Cali, Colombia; ejrbaron@gmail.com

2. Laboratorio de Histotecnia, Escuela de Biología, Universidad Industrial de Santander, Carrera 27 con calle 9 , Bucaramanga,Colombia; vivigela@gmail.com, helfos85@gmail.com

3. Unidad de Microscopia Electrónica, Carrera 2 \# 1A-25, Urbanización Caldas, Universidad del Cauca, Popayán, Colombia; gantorres@gmail.com

4. Laboratorio de Estudios de Anatomía Vegetal Evolutiva y Sistemática (LEAVES), Facultad de Ciencias Naturales y Museo, Universidad Nacional de La Plata, 64 entre 120 y diagonal 113, B1904 DZB, La Plata, Argentina; tinar.cris@ gmail.com,tinar@speedy.com.ar

5. Consejo Nacional de Investigaciones Científicas y Técnicas (CONICET), Buenos Aires, Argentina.

6. Grupo Microbiota, Campus Universitario Lagos del Cacique, Universidad de Santander (UDES), Bucaramanga, Santander, Colombia.

Recibido 14-XII-2010. Corregido 30-III-2011. Aceptado 28-IV-2011.

\begin{abstract}
Ontogeny of strobili, sporangia development and sporogenesis in Equisetum giganteum (Equisetaceae) from the Colombian Andes. Studies on the ontogeny of the strobilus, sporangium and reproductive biology of this group of ferns are scarce. Here we describe the ontogeny of the strobilus and sporangia, and the process of sporogenesis using specimens of E. giganteum from Colombia collected along the Rio Frio, Distrito de Sevilla, Piedecuesta, Santander, at $2200 \mathrm{~m}$ altitude. The strobili in different stages of development were fixed, dehydrated, embedded in paraffin, sectioned using a rotatory microtome and stained with the safranin $\mathrm{O}$ and fast green technique. Observations were made using differential interference contrast microscopy (DIC) or Nomarski microscopy, an optical microscopy illumination technique that enhances the contrast in unstained, transparent. Strobili arise and begin to develop in the apical meristems of the main axis and lateral branches, with no significant differences in the ontogeny of strobili of one or other axis. Successive processes of cell division and differentiation lead to the growth of the strobilus and the formation of sporangiophores. These are formed by the scutellum, the manubrium or pedicel-like, basal part of the sporangiophore, and initial cells of sporangium, which differentiate to form the sporangium wall, the sporocytes and the tapetum. There is not formation of a characteristic arquesporium, as sporocytes quickly undergo meiosis originating tetrads of spores. The tapetum retains its histological integrity, but subsequently the cell walls break down and form a plasmodium that invades the sporangial cavity, partially surrounding the tetrads, and then the spores. Towards the end of the sporogenesis the tapetum disintegrates leaving spores with elaters free within the sporangial cavity. Two layers finally form the sporangium wall: the sporangium wall itself, with thickened, lignified cell walls and an underlying pyknotic layer. The mature spores are chlorofilous, morphologically similar and have exospore, a thin perispore and two elaters. This study of the ontogeny of the spore-producing structures and spores is the first contribution of this type for a tropical species of the genus. Fluorescence microscopy indicates that elaters and the wall of the sporangium are autofluorescent, while other structures induced fluorescence emitted by the fluorescent dye safranin $\mathrm{O}$. The results were also discussed in relation to what is known so far for other species of Equisetum, suggesting that ontogenetic processes and structure of characters sporoderm are relatively constant in Equisetum, which implies important diagnostic value in the taxonomy of the group. Rev. Biol. Trop. 59 (4): 1845-1858. Epub 2011 December 01.
\end{abstract}

Key words: Equisetum, strobilous, sporangium ontogeny, sporogenesis, spores, autofluorescence, induced fluorescence. 
Las Equisetaceae son cosmopolitas. Incluyen un solo género Equisetum L., que reúne unas 15 especies, y esta dividido en dos subgéneros, Equisetum, que incluye: E. arvense L., $E$. bogotense Kunth., E. diffusum D. Don, E. fluviatile L., E. palustre L., E. pratense Ehrh., E. sylvaticum L. y E. telmateia Ehrh. e Hippochaete (J. Milde) Baker (Hauke 1963, 1978, 1995), en el que se agrupan: E. giganteum L., E. myriochaetum Schltr. \& Cham., E. hyemale L., E. laevigatum L., E. ramosissimum Desf., E. scirpoides Michx. y E. variegatum Schleich.

Los estudios realizados sobre la morfología y los análisis moleculares de ADN sobre secuencias plastidiales $r b c L, t r n L-F, r p s 4$ sugieren que es monofilético (Pryer et al. 2001, 2004, Des Marais et al. 2003, Guillon 2004, Schuettpelz et al. 2006, Schuettpelz \& Pryer 2008). Esos estudios también señalan que representaría un clado hermano de los helechos eusporangiados (Marattiales) y de los helechos leptosporangiados. Los tres grupos integrarían, juntos, un clado hermano de Ophioglossaceae y Psilotaceae (Pryer et al. 2001, 2004). De acuerdo con la morfología de los espermatozoides (Renzaglia et al. 2000) y los caracteres de las raíces (Kato 1983), se sitúan, con el resto de los helechos, en las Monilophyta, un grupo de reciente concepción y aún en revisión, basado en las Moniliformopses, nombre originalmente propuesto por Kenrick \& Crane (1997) que se fundamenta en caracteres morfológicos tales como la presencia de raíces caulógenas originadas en la endodermis, protoxilema mesarco, tapete plasmodial, espermatozoides pluriflagelados y caracteres genéticos, como la inserción de nueve nucleótidos en el gen plastídico rps4.

Los equisetos tienen un patrón distintivo de crecimiento en nudos y entrenudos, tallos verticilados y hojas reducidas, connatas, en verticilos regulares (Tryon \& Tryon 1982, Moran 1995). Los tallos tienen estructura interna lacunar, tejidos vasculares fragmentados en cordones con lagunas protoxilemáticas y áreas centrales huecas. Las estructuras esporógenas están reunidas en estróbilos terminales. Cada estróbilo tiene un eje central portador de esporangióforos, estructuras peltadas con una porción basal que se asemeja a un pedicelo, el manubrio, y una porción apical aplanada, el escutelo (a veces llamada vulgarmente escudo). Cada esporangióforo lleva un anillo de esporangios péndulos dirigidos hacia el eje del estróbilo.

Las esporas son clorofílicas, presentan una única abertura circular que no se encuentra en otros helechos, están cubiertas por un perisporio delgado, casi membranoso, de esporopolenina, que se adosa en la zona de abertura y cubre por completo la espora y se caracterizan por la presencia de dos apéndices en forma de banda o cinta, higroscópicos, los eláteres o elaterios unidos a la superficie de esporas en un punto común (Hauke 1963, Lugardon 1978, Tryon \& Lugardon 1990). Los estudios más recientes sugieren que las paredes esporales también contienen sílice (Currie \& Perry 2009).

Tres especies, E. bogotense Kunth., E. giganteum L. y E. myriochaetum Schltdl. \& Cham., se citan para Centroamérica y Sudamérica (Moran 1995), y crecen también en Colombia (Triana \& Murillo 2005). Según Tryon \& Tryon (1982) E. giganteum vive en América tropical, desde el sur de México hasta la Argentina; al igual que las otras dos especies mencionadas para Colombia, tiene amplia distribución; es una planta predominantemente palustre, tanto de ambientes lóticos como lénticos y humedales; tanto en zonas despejadas como áreas más protegidas como orillas de quebradas y bordes de barrancos sombríos y húmedos. El rango altitudinal de las tres especies es similar, desde los 900 hasta los $4500 \mathrm{~m}$ (Murillo \& Harker 1990), aunque E. giganteum es más común en zonas más bajas, entre los 600 y $3000 \mathrm{~m}$ de altitud (Triana \& Murillo 2005).

Los trabajos recientes en el género Equisetum son abundantes, de carácter taxonómico, sistemático y evolutivo (Pryer et al. 1995, Renzaglia et al. 2000, Pryer et al. 2001, Des Marais et al. 2003, Guillon 2004, 2007, Smith et al. 2006). Otros trabajos tratan el género en relación con su capacidad de biomineralizar sílice y acumularla en diversas partes del cuerpo vegetal, uno de sus caracteres más notables, así 
como la importancia de este compuesto biogenerado en la fisiología de las plantas (Kaufman et al. 1973, Chen \& Lewin 1969, Ernst 1990, Epstein 1999, Holzhuter et al. 2003, Currie \& Perry 2007, Currie \& Perry 2009).

Los estudios de la biología reproductiva de las equisetáceas, en cambio, son escasos. Hawkins (1907) describió el desarrollo de los esporangios y la esporogénesis de E. hyemale, Beer (1909) analizó el proceso de maduración de las esporas de E. arvense y E. fluviatile L. (sub. E. limosum L), registró la presencia de clorofila y describió el patrón de depósito de las capas del esporodermo. Page (1972) estudió la morfología y evolución de los esporangióforos y ejes para determinar el origen de la disposición de estas estructuras. Gullvăg (1968) y Olsen \& Gullvăg (1973) estudiaron algunos aspectos ultraestructurales relacionados con la germinación de las esporas en E. fluviatile y E. arvense respectivamente. Duckett (1970) relacionó la forma, tamaño y color de las esporas de Equisetum con su viabilidad, y señaló las diferencias que se pueden encontrar entre las especies fértiles y los híbridos. Posteriormente, Lehmann et al. (1984), detallaron algunos caracteres estructurales y ultraestructurales de la esporogénesis de E. fluviatile. Uehara \& Kurita (1989) describieron aspectos ultraestructurales hallados en el curso de la esporogénesis, así como la morfogénesis de la pared de las esporas y los eláteres de E. arvense; esos autores señalaron además que la maduración de los esporangios en los estróbilos es basípeta, por lo que es posible encontrar todas las etapas del proceso de formación de las esporas en un estróbilo maduro. Tryon \& Lugardon (1990) estudiaron la ultraestructura de los eláteres y la pared de las esporas de varias especies de Equisetum. Uehara \& Murakami (1995) observaron la disposición de los microtúbulos del tapete durante el proceso de formación de las esporas y mostraron la formación de tapetal gaps (aquí llamadas cámaras plasmodiales), alrededor de las tétradas y posteriormente alrededor de las esporas en formación. Finalmente, Roshchina et al. (2004), evaluaron la utilidad de la técnica de microscopia confocal en el estudio de la fisiología de la espora, con la observación de la autofluorescencia en esporas de $E$. arvense.

A pesar de que se cuenta con información general sobre la esporogénesis de algunas especies de Equisetum (Gullvăg 1968, Olsen \& Gullvắg 1973, Tryon \& Lugardon 1990), hasta el momento no hay trabajos que incluyan una descripción detallada de la ontogenia de los estróbilos, esporangios y esporogénesis en especies del género. A esto debe agregarse que las referencias existentes corresponden a especies de zonas templadas y no hay estudios sobre estos procesos en especies tropicales.

Por lo que antecede, este trabajo se propone describir la ontogenia de los estróbilos, los esporangios y efectuar aportes al conocimiento del proceso de esporogénesis de $E$. giganteum, con datos nuevos y originales sobre la biología reproductiva de esta especie. Los resultados obtenidos se discuten en relación con lo conocido hasta el momento para otras especies de Equisetum.

\section{MATERIALES Y MÉTODOS}

Durante la época seca se recolectaron 30 ejemplares de E. giganteum, entre los meses de enero y febrero de 2010, a orillas del Río Frío en las inmediaciones de la Estación Experimental El Rasgón, de la Corporación Autónoma Regional para la Defensa de la Meseta de Bucaramanga (CDMB), en la vereda Cristales, Corregimiento de Sevilla, Municipio de Piedecuesta, Santander a $7^{\circ} 2^{\prime} 29^{\prime \prime}$ N, $72^{\circ} 57^{\prime} 32^{\prime \prime} \mathrm{W}$ y $2200 \mathrm{~m}$ de altitud. El material de soporte de este trabajo fue incluido en el Herbario de la Escuela de Biología de la Universidad Industrial de Santander (UIS) referido a un número de muestra con la clave EG-015.

Los estróbilos del eje principal y las ramificaciones fueron clasificados en siete etapas de maduración, de las cuales se tomaron diez estróbilos por cada una de estas etapas. El material recolectado se fijó en FAA (formolalcohol-acido acético) durante 24h. Posteriormente, se cortaron en fragmentos de $1 \mathrm{~cm}$ de longitud para deshidratarlos en la serie habitual de alcoholes y dos pasos de aclaramiento en 
xilol (Ruzin 1999). Los fragmentos fueron embebidos en Paraplast (Mc Cormick ${ }^{\circledR}$ ) durante $12 \mathrm{~h}$ a $55^{\circ} \mathrm{C}$. Se obtuvieron secciones longitudinales y transversales con un micrótomo rotatorio Spencer 820, en espesores de 3-5 $\mu \mathrm{m}$ y las secciones obtenidas se colorearon con safranina O-fast green (Johansen 1940). Las muestras se examinaron con un microscopio fotónico de alta resolución Nikon 80i Eclipse, equipado con DIC y un microscopio Nikon Eclipse e200 equipado con fluorescencia, se utilizó filtro B-2 (filtro de excitación 450$490 \mathrm{~nm}$ y filtro barrera $515 \mathrm{~nm}$ ). Las fotografías de alta resolución y fluorescencia se obtuvieron con cámara digital Nikon DS-2MV y cámara Canon PowerShot A710 IS respectivamente, en la Unidad de Microscopía Electrónica de la Universidad del Cauca y el laboratorio de Morfología Vegetal de la Universidad del Valle. Todo el procesamiento del material se llevó a cabo en el Laboratorio de Histotecnia de la Escuela de Biología de la Universidad Industrial de Santander.

Los términos pteridológicos utilizados en este trabajo se encuentran en Lellinger (2002). De modo que se ha introducido en español el término cámaras plasmodiales para designar las formaciones que Uehara \& Kurita (1989) llamaron tapetal gaps por considerárselo apropiado tanto en los descriptivo como funcional, y así evitar la traducción literal.

\section{RESULTADOS}

Los estróbilos maduros del eje principal de E. giganteum alcanzan una longitud de $3( \pm 0.5) \mathrm{cm}$ (Fig. 1), y los de las ramas laterales de $1.7( \pm 0.7) \mathrm{cm}$. No se observaron diferencias en la ontogenia y la esporogénesis entre los estróbilos del eje principal y los de las ramas laterales, y se pudo constatar que este proceso es sincrónico, puesto que todos los esporangios en un estróbilo, presentan el mismo grado de maduración.

La formación de los estróbilos se inicia a partir de tejido parenquimático derivado del meristemo apical. Las células meristemáticas son isodiamétricas y presentan un núcleo voluminoso. En el tejido meristemático se observan las áreas correspondientes a los nudos y entrenudos de los ejes aéreos, así como las hojas se encuentran formando verticilos alrededor de los nudos (Fig. 2).

A medida que se desarrollan y crecen los estróbilos, se observa la formación de grupos de células parenquimáticas en todo el contorno de sus ejes. Estas células dan origen a los esporangióforos (Fig. 3). Cerca de la base de los esporangióforos, longitudinalmente y en toda la extensión del eje del estróbilo aparecen grupos de células parenquimáticas más o menos conectados entre sí. Las células de estos grupos se alargan, se diferencian y en su fase más avanzada forman engrosamientos helicoidales, dando origen a grupos de traqueidas que se proyectan hacia el esporangióforo, y contribuyen a la vascularización de los esporangios (Figs. 4-6).

Los agregados celulares que formarán los esporangios se caracterizan por sus células con citoplasma abundante y núcleos voluminosos marcadamente eucromáticos (Fig. 7). Estas células dan origen a la pared del esporangio, de varias capas celulares y al tejido esporógeno (Fig. 8). Este último, por activas mitosis, forma rápidamente los esporocitos y las células del tapete que en esta etapa está constituido, generalmente, por un estrato de células de citoplasma escaso (Fig. 9). Los esporocitos tienen forma variable y contorno poligonal, núcleo altamente eucromático con varios nucléolos y citoplasma de aspecto granular/fibrilar (Fig. 10). A medida que maduran presentan un comportamiento nuclear que se caracteriza por una alta condensación de la cromatina en una región de la periferia del núcleo, en tanto que la membrana nuclear permanece intacta. Asimismo, se observan estructuras filamentosas que conectan este material condensado y la membrana nuclear. Los nucléolos son visibles y el citoplasma presenta una apariencia filamentosa (Fig. 11).

A medida que crecen los esporangióforos, se produce la formación del escutelo y el eje del esporangióforo o manubrio se hace más prominente (Fig. 12). 

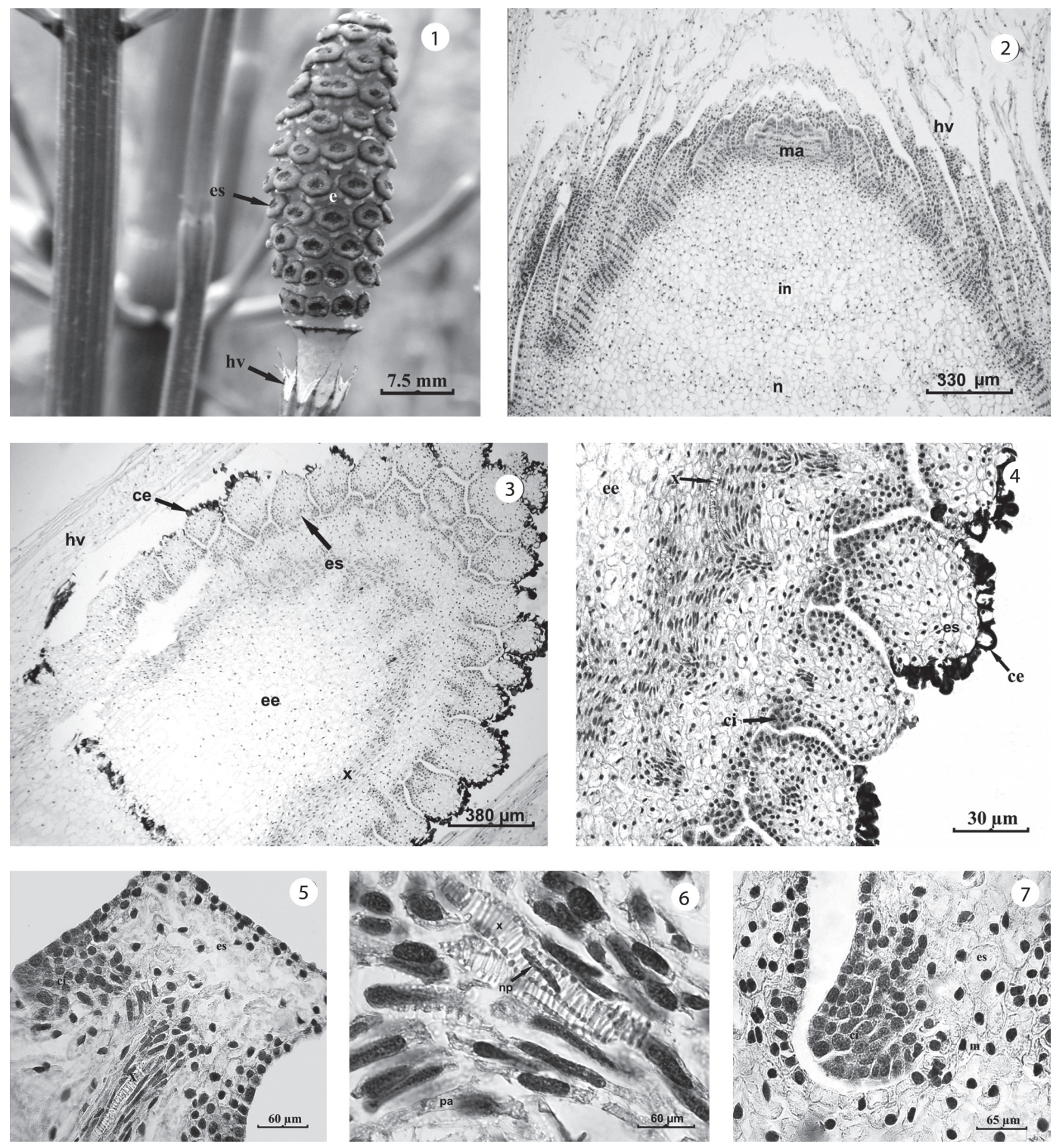

Figs. 1-7. Estróbilo maduro, etapas tempranas de la ontogenia del estróbilo y esporangióforos de E. giganteum. 1. Estróbilo maduro del eje principal, con esporangióforos densamente agrupados a lo largo del eje estrobilar. 2. Zona meristemática que formará el estróbilo. 3-6. Desarrollo del estróbilo, formación de los esporangióforos y los esporangios; se observa el crecimiento de las estructuras por actividad mitótica y diferenciación celular.7. Esporangióforo con células iniciales del esporangio. ce: células del escutelo; ci: células iniciales del esporangio; ee: eje del estróbilo; m: manubrio; es: esporangióforo; hv: hojas verticiladas; in: entrenudo; ma: meristemo apical; n: nudo; np: núcleo picnótico; pa: parenquima; $\mathrm{x}$ : xilema.

Figs. 1-7. Adult strobilus, early stages of the ontogeny of strobilous and the sporangiophores of E. giganteum. 1. Adult strobilus main axis, with sporangiophores densely clustered along the strobilar axis. 2. Meristematic region that will form the strobilus. 3-6. Development of strobilus, formation of sporangiophores and sporangia; the active growing of structures through mitotic activity and differentiation of cells is observed.7. Sporangiophore with sporangium initials. ce: scutellum cells; ci: sporangium initials cells; ee: strobilus axis; m: manubrium; es: sporangiophore; hv: verticillate leaves, in: internode, ma: apical meristem; n: node; np: pyknotic nucleus; pa: parenchyma; x: xylem. 
El escutelo está formado por tejido parenquimático y por una epidermis que madura rápidamente: sus células adultas presentan una pared externa formada por una delgada capa celulósica interna, brillante, que no se colorea y capas cuticulares de $10-15 \mu \mathrm{m}$ de espesor, marcadamente coloreadas. La cutinización se extiende a las paredes anticlinales, y las excede con el aspecto de cuñas cuticulares de tal forma que alcanza parte de las paredes tangenciales internas. La pared externa de la epidermis de los esporangióforos adultos presenta abundantes verrugas cuticulares. Paralelamente, se produce contracción del citoplasma y picnosis nuclear (Fig. 13).

$\mathrm{Al}$ iniciarse la meiosis en los esporocitos, el tapete pierde la integridad histológica e invade parcialmente los espacios entre estos, al adquirir una estructura plasmodial e iniciar la formación de las cámaras plasmodiales, las cuales reúnen agrupaciones de tétradas (Fig. 14). Es probable que la etapa de tétradas transcurra rápidamente, debido a que no se observa con frecuencia en las secciones obtenidas.

Al avanzar la maduración del esporangio, las proyecciones del tapete plasmodial rodean las esporas en formación, las mismas quedan incluidas en cámaras plasmodiales individuales; estas cámaras están delimitadas internamente por el esporodermo en desarrollo y externamente por la membrana del plasmodio (Fig. 15-16). En las cámaras plasmodiales se desarrolla el perisporio y posteriormente los eláteres (Fig. 16). Finalmente, el tapete degenera y las esporas con sus eláteres quedan libres en la cavidad esporangial (Fig. 17).
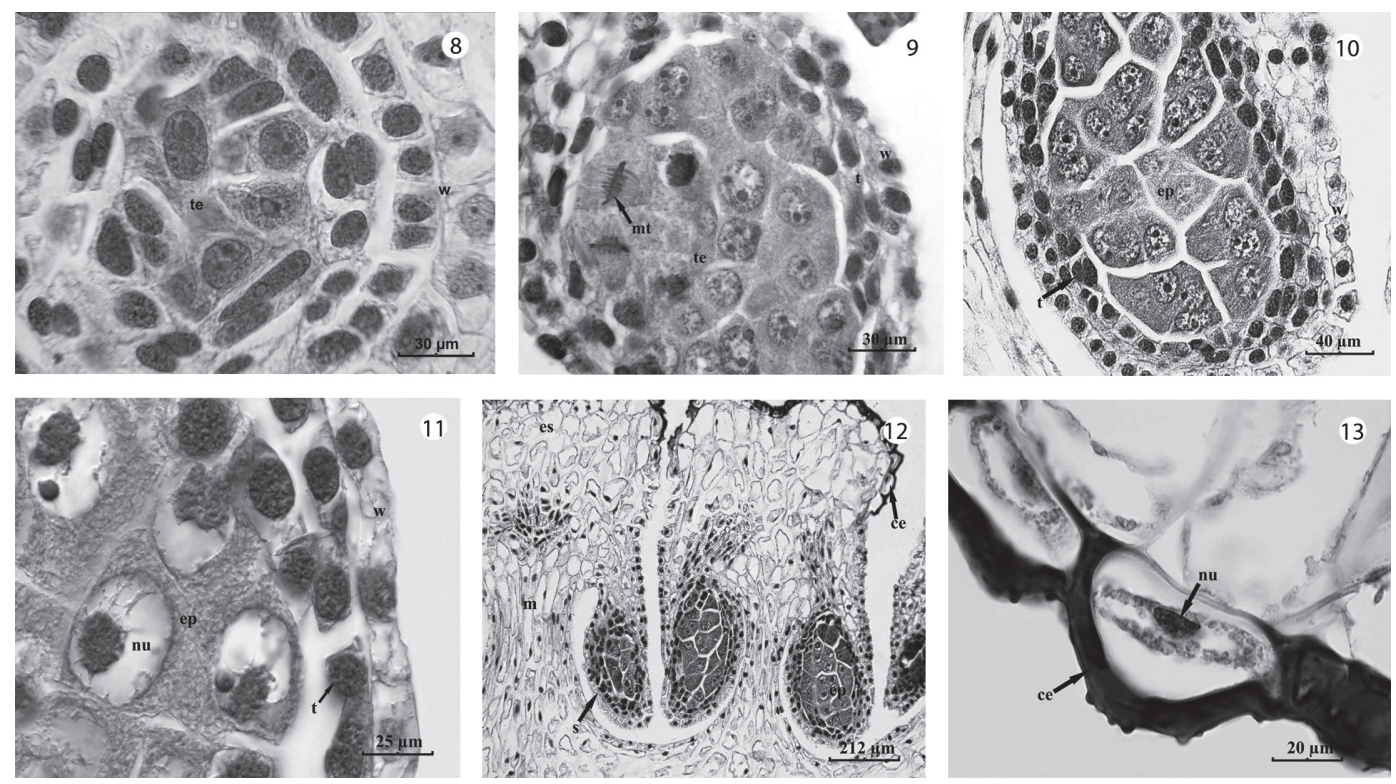

Figs. 8-13. Esporangióforo y esporangios de E. giganteum, etapas iniciales y caracteres anatómicos. 8-9. Comienzo de la diferenciación del tejido esporógeno, tapete y pared del esporangio. 10. Esporangio, esporocitos y tapete celular. 11. Esporocitos premeióticos. 12. Corte longitudinal del esporangióforo; engrosamientos de la epidermis del escutelo. 13. Epidermis del escutelo, engrosada y verrucosa. ce: células del escutelo; ep: esporocitos; es: esporangióforo; m: manubrio; mt: mitosis; nu: núcleo; s: esporangio; t: tapete; te: tejido esporógeno; w: pared del esporangio.

Figs. 8-13. Sporangiophore and sporangia of E. giganteum, early stages and anatomical characters. 8-9. Beginning of the differentiation of sporogenous tissue, tapetum and sporangium wall. 10. Sporangium, sporocytes, and tapetum in a celular stage. 11. Pre-meiotic sporocytes.12. Longitudinal section of the sporangiophore; thickening epidermis of the scutellum. 13. Thickened, verrucose epidermis of scutellum. ce: scutellum cells; ep: sporocytes; es: sporangiophore; m: manubrium; mt: mitosis; nu: nucleus; s: sporamgium; t: tapetum; te: sporogenous tissue; w: sporangium wall. 
Para esta misma etapa, la pared del esporangio consta de un estrato celular externo y otro interno. El externo constituye la pared definitiva del esporangio y está constituido por células que presentan engrosamientos en las paredes anticlinales y periclinales internas, aunque es posible observar también varias células con engrosamientos lignificados en la pared periclinal externa. El estrato celular interno se caracteriza por la presencia de células de citoplasma escaso con núcleos alargados (Fig. 18).

Las esporas maduras se encuentran recubiertas por el perisporio, una fina capa de esporopolenina que se adhiere a la zona de abertura de la espora (Figs. 18-19). La región de la abertura esporal es biconvexa y localizada en el sitio de unión de las esporas en la tétrada, es decir, en la cara proximal de la espora; en este mismo sitio se adosan los eláteres. El núcleo de la espora presenta contorno irregular, se ubica en posición central, presenta un nucléolo prominente y el citoplasma aparece granular (Fig. 19).

En las observaciones con microscopía de fluorescencia, las esporas y los eláteres emiten fluorescencia en el rango de longitud de onda del amarillo-naranja; las células de la pared definitiva del esporangio y las epidérmicas del escutelo, emiten fluorescencia amarilla mientras que las demás células del escutelo, las parenquimáticas del estróbilo, los núcleos y los nucléolos de las esporas, emiten fluorescencia roja (Fig. 20).

\section{DISCUSIÓN}

Aquí se han estudiado los procesos de desarrollo de los estróbilos, esporangióforos, esporangios y esporas en la especie neotropical E. giganteum. La ontogenia de las estructuras esporógenas y las esporas es el primer aporte de este tipo para una especie tropical del género. El desarrollo de los estróbilos es similar en todos los ejes; los esporangióforos se diferencian rápidamente y no forman un arquesporio típico, la esporogénesis es sincrónica, el tapete es histológicamente discreto hasta que se produce la meiosis de los esporocitos y en esa etapa cambia a un estado plasmodial, invade la cavidad esporangial y rodea parcialmente las tétradas de esporas, y da origen a cámaras plasmodiales en las que se depositan materiales estructurales, formadores de pared (exosporio y perisporio) de las esporas. La microscopía de fluorescencia indica que los eláteres y la pared del esporangio son autofluorescentes, en tanto que otras estructuras emiten fluorescencia inducida por el colorante safranina $\mathrm{O}$.

En relación con el desarrollo de los estróbilos, Hawkins (1907), en estudios realizados sobre $E$. hyemale señaló que se inician a partir de células meristemáticas y por divisiones anticlinales y periclinales forman grupos de células parenquimáticas los cuales se diferenciarán luego en los tejidos adultos de los estróbilos. Esos grupos originan masas pluricelulares sobresalientes, dispuestas helicoidalmente sobre el eje del estróbilo, a partir de las cuales se forman los esporangióforos. Esto coincide con lo observado en E. giganteum y también en E. bogotense (Rincón et al., datos no publicados). Las escasas referencias bibliográficas sobre este tema impiden generalizar la hipótesis sobre si este modelo de desarrollo de los esporangióforos es similar y puede generalizarse a otras especies de Equisetum.

En E. giganteum, los grupos de células parenquimáticas en los esporangióforos experimentan divisiones periclinales y anticlinales para originar los esporangios y generan dos estratos celulares: uno interno de crecimiento rápido que corresponde al tejido esporógeno y otro externo, de crecimiento lento, que origina la pared del esporangio. El tejido esporógeno se diferencia rápidamente para formar directamente los esporocitos y el tapete. Aunque, aparentemente esto es similar a lo descrito por Hawkins (1907) para E. hyemale y por Uehara \& Kurita (1989) para E. arvense, esos autores destacan que el tejido esporógeno pasa por una fase de arquesporio previa a la formación de los esporocitos y el tapete, una condición que no se observó en E. giganteum, especie en la que no se halló tejido arquespórico.

En relación con el proceso de esporogénesis, Lehmann et al. (1984) lo consideraron 

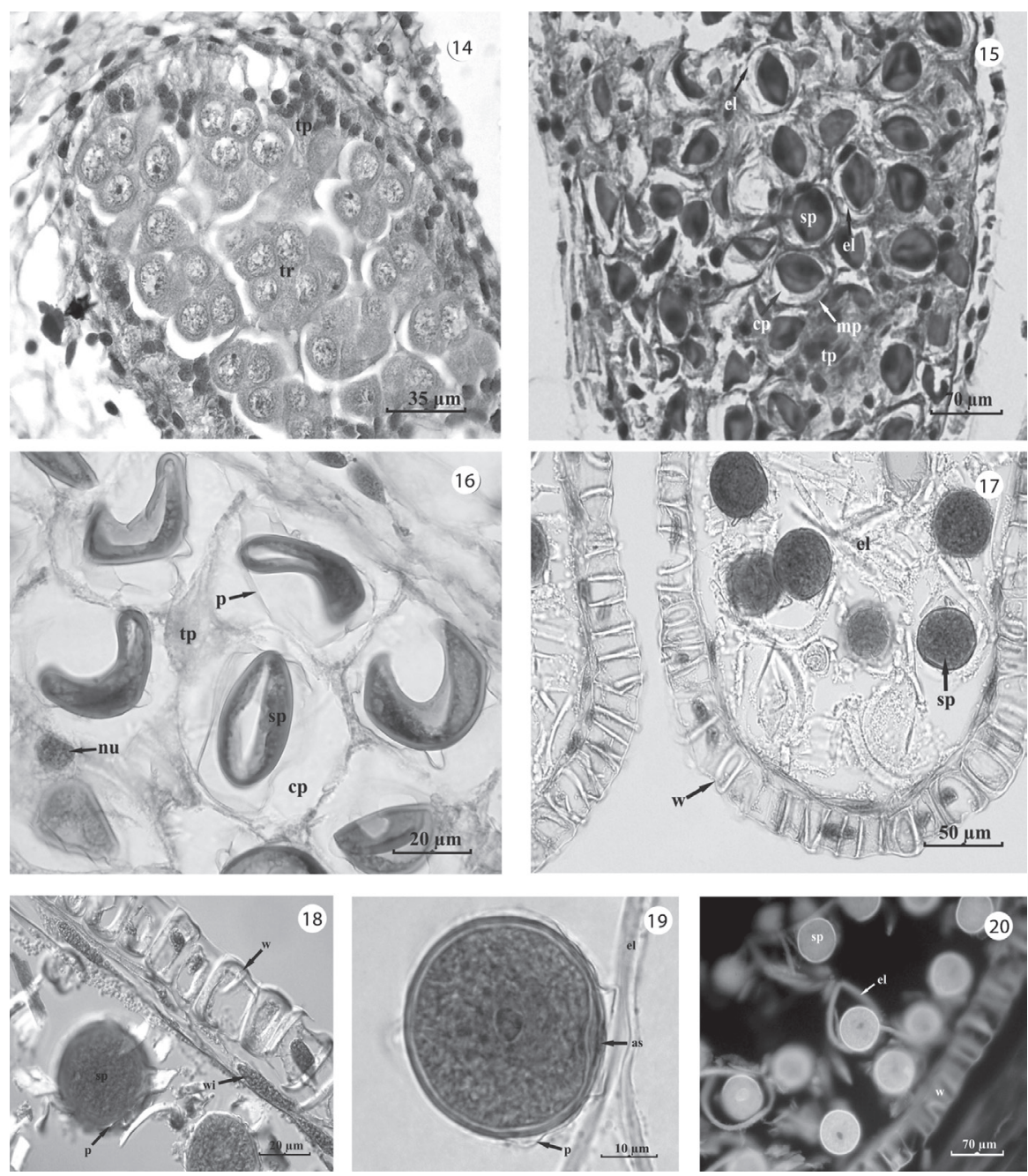

Figs. 14-20. Esporangio y esporas de E. giganteum. 14. Tétradas de esporas en grupos regulares dentro del esporangio; invasión del tapete plasmodial hacia la cavidad esporangial. 15-16. Tapete plasmodial, formación de cámaras plasmodiales alrededor de cada espora; eláteres y perisporio en formación en el interior de las cámaras plasmodiales. 17. Pared del esporangio maduro y esporas con eláteres. 18. Detalles de las capas interna y externa de la pared del esporangio, la externa con engrosamientos en paredes anticlinales y periclinales; esporas con perisporio y eláteres. 19. Relación del perisporio con el área de la abertura esporal. 20. Autofluorescencia amarillo/naranja emitida por el esporodermo, eláteres y pared del esporangio con fluorescencia roja inducida, emitida por núcleos, nucléolos y células parenquimáticas. as: abertura esporal; cp: cámaras plasmodiales; el: eláteres; mp: membrana plasmodial; nu: núcleo del tapete plasmodial; p: perisporio; sp: esporas; tp: tapete plasmodial; tr: tétradas; w: pared externa del esporangio; wi: pared interna del esporangio.

Figs. 14-20. Sporangium and spores of E. giganteum. 14. Tetrads of spores in regular clusters within the sporangium; invasion of plasmodial tapetum to the sporangial cavity.15-16. Tapetum surrounding the spores in plasmodial chamber; development of elaters and perispore within tapetal gaps. 17. Mature sporangium wall and spores with elaters. 18. Details of the inner and outer layer of the sporangium wall, the outer with thickened anticlinal and periclinal cell walls; spores with perispore and elaters. 19. Perispore position in relation to the spore aperture. 20. Yellow / orange autofluorescence emitted by the sporangium wall, the sporoderm, and the elaters; red induced fluorescence emitted by nuclei, nucleoli and parenchyma cells. as: opening spore; cp: plasmodial chamber; el: elaters; mp: plasmodial membrane; nu: nucleus of plasmodial tapetum; p: perispore; sp: spores; tp: plasmodial tapetum; tr: tetrads; w: outer wall of the sporangium; wi: inner wall of the sporangium. 
asincrónico en E.fluviatile, mientras que Uehara \& Kurita (1989), lo describieron como basípeto al estudiar el desarrollo de los estróbilos de E. arvense, e indicaron que existe la posibilidad de encontrar todas las etapas del proceso de esporogénesis en un mismo estróbilo maduro. En el caso de E. giganteum se observó que la formación y maduración de las esporas es sincrónico, una condición similar a la de $E$. arvense y observada también en E. bogotense (Rincón et al., datos no publicados).

Los esporocitos de E. giganteum presentan un núcleo altamente eucromático, con uno o varios nucléolos, similar a lo observado por Lehmann et al. (1984) y Uehara \& Kurita (1989) para E. fluviatile y E. arvense respectivamente, aunque estos autores no mencionan la apariencia fibrilar en el citoplasma que se observó en E. giganteum.

Los esporocitos de E. giganteum atraviesan por sucesivas etapas de maduración que no han sido registrados para otras especies de Equisetum: inicialmente se dividen activamente por mitosis y aumentan rápidamente en cantidad; luego, en etapas premeióticas el material nuclear se condensa en un área del nucleoplasma, mientras que la membrana nuclear permanece intacta y se forman estructuras de apariencia fibrilar entre el material condensado y la envoltura nuclear. El fenómeno descrito se atribuiría al deterioro natural de las células en el curso de su diferenciación, y no a las técnicas de procesamiento de las muestras, ya que se observó coexistencia de células colapsadas y normales. Dado que estas observaciones fueron realizadas mediante microscopio de luz, un próximo estudio contempla analizar el proceso descrito con microscopía electrónica de transmisión (MET), con el fin de aclarar este aspecto. Las estructuras de apariencia fibrilar también han sido registradas en musgos (Karasawa 1986), helechos (Sheffield et al. 1983), y plantas superiores (Sheffield et al. 1979, Majewska- Sawka et al.1990) al parecer en relación con la formación de vacuolas nucleares.

El tapete es una estructura que se relaciona siempre con los procesos de esporogénesis
(Lugardon 1990, Pacini \& Franchi 1993, Parkinson \& Pacini 1995, Pacini 1997, Furness \& Rudall 2001, Furness 2008). En Equisetum giganteum, es inicialmente celular pero se transforma plasmodial e invade los espacios entre los esporocitos, cuando éstos inician la meiosis. Esta condición es similar a la descrita para otras especies de Equisetum (Uehara \& Kurita 1989, Lugardon 1990, Uehara \& Murakami 1995).

Al finalizar la meiosis, las tétradas se encuentran parcialmente rodeadas por el tapete plasmodial y se aprecian agrupaciones de éstas en la cavidad del esporangio. Similares características de las tétradas y el tapete fueron registradas, en estudios efectuados con MET, por Uehara \& Kurita (1989), no obstante esos autores señalan que en esa etapa de maduración el tapete rodea totalmente las tétradas. En este trabajo se pudo constatar que el tapete invade completamente la cavidad del esporangio sólo hasta que las esporas se han liberado de las tétradas y luego éstas quedan encerradas en el interior de cámaras plasmodiales individuales. Los movimientos del tapete y la formación de las cámaras plasmodiales son muy similares a los descritos por Parkinson (1987) para Psilotum nudum (L.) P. Beav. Aunque el término cámara plasmodial no ha sido utilizado previamente en relación con Equisetum, su formación, comportamiento y función indica que es equivalente a lo que se designa en lengua inglesa como "tapetal gap". En este trabajo se ha preferido introducir ese término en lugar de una traducción literal del inglés. Hacia el final de la esporogénesis de E. giganteum se pueden observar ya formados los eláteres y el perisporio, todavía en el interior de las cámaras plasmodiales, algo que es coincidente con $E$. arvense (Uehara \& Kurita 1989, Uehara \& Murakami 1995). Las imágenes efectuadas mediante microscopía de luz y MET son coincidentes, si bien las tomadas con la segunda técnica permiten refinar estas observaciones.

Una vez que el tapete se desintegra y degenera, sólo se observa una capa de células parietales de apariencia picnótica que cubren la cavidad del esporangio, un carácter que 
también se registró para E. bogotense (Rincón et al., datos no publicados), pero no para otras especies de Equisetum. Las células de la pared del esporangio maduro de E. giganteum presentan diferentes grados de engrosamiento en todas las paredes celulares; este carácter difiere de la mayoría de licófitos y otros monilófitos, que presentan engrosamientos de las paredes del esporangio en forma de "U" (Øllgaard 1975, Rolleri 1972, Uehara \& Kurita 1991, Moran 2004, Rincón et al. 2009). Estos engrosamientos actúan como un mecanismo sencillo para catapultar a las esporas, de manera que ayudarían en la dispersión. En las esporas de Equisetum, la dispersión estaría basada en la presencia y eficiencia de los eláteres (Pacini \& Franchi 1993).

En relación con las esporas de Equisetum, no existe un acuerdo generalizado en la bibliografía acerca de los términos aplicables a las capas de la pared. Uehara \& Murakami (1995) describen la pared de las esporas de Equisetum como formada por cuatro capas: intina, exina, capa media y eláteres, e indican que la exina se forma después de la meiosis y consiste en dos capas distintas, una interna y otra externa, la cual se deposita luego de la formación de la capa interna; lo que esos autores llaman capa media o intermedia, se deposita después de la exina. Los términos intina y exina son típicos de los granos de polen y por lo tanto, se descartan en este trabajo y no deberían usarse en relación con esporas. El perisporio es la capa externa que proviene de la desintegración del tapete, mientras que se define como el episporio la capa externa de esporopolenina, que se encuentra por fuera del exosporio en algunos helechos heterosporados y en Equisetum (Lellinger, 2002). Tryon \& Lugardon (1990) llaman episporio a las capas de esporopolenina externas al exosporio en Marsileaceae, Azolla y Salvinia. El término perina (Erdtman 1943, Perkins et al.1985) se utiliza a veces como sinónimo de perisporio, pero Punt et al. (2007) consideran que el término perina no debe usarse en relación con esporas, y sí en conjunto con sexina y nexina, para designar capas de pared en los granos de polen, en tanto que perisporio debe usarse conjuntamente con exosporio y endosporio, siempre en relación con la pared esporal. Por otra parte debe considerarse el origen de estas capas: el exosporio proviene del protoplasto de las esporas, en tanto que las otras capas citadas, independientemente de su nombre, se originan a partir del tapete, indistintamente de su condición celular o plasmodial (Beer 1909 y autores posteriores). En este sentido, el origen del perisporio y el episporio serían coincidentes, aunque difieran sus nombres y aún podrían usarse indistintamente. Aquí se ha preferido utilizar el término perisporio.

Las esporas de E. ramosissimum, E. arvense y E. palustre han sido descritas por Uehara \& Kurita (1989) y Lugardon (1990). Así mismo, Tryon \& Lugardon (1990) ilustran las esporas de E. bogotense, E. debile Roxb., E. giganteum, E. hyemale y E. telmateia. Las esporas de estas especies son esféricas, clorofílicas, con dos eláteres, presentan el perisporio unido a la zona de la abertura esporal, que tiene un engrosamiento de forma lenticular, caracteres que también se pueden atribuir a E. giganteum.

La fluorescencia amarillo/naranja que se vio en el esporodermo de E. giganteum es inducida, producto de las propiedades fluorescentes de la safranina que colorea intensamente la esporopolenina. Los resultados aquí obtenidos coinciden con lo observado por Ruzin (1999) y Haseloff (2003) en relación con este colorante y componentes de los tejidos vegetales, aunque Ruzin (1999) indica que la esporopolenina emite autofluorescencia amarilla/roja sólo cuando es excitada con luz ultravioleta. Los núcleos y los nucléolos de las esporas y de las células de la pared del esporangio en $E$. giganteum emiten fluorescencia roja; en este caso también se interpreta como inducida por la coloración con safranina, dado que la cromatina no sería autofluorescente (Ma et al. 1993, Ruzin 1999).

Los eláteres muestran fluorescencia amarillo/naranja, similar a la observada en la pared de las esporas. Los eláteres se colorean intensamente con fast green, un colorante que no induce fluorescencia (Bond et al. 2008), 
algo que sugiere que en éstos se trataría de autofluorescencia, que provendría de algún compuesto presente en estas estructuras. Tryon \& Lugardon (1990) señalan que están formados por dos capas: una interna, delgada, constituida principalmente de celulosa y otra externa, de mayor espesor, formada por polisacáridos no celulósicos. En estos componentes habría que buscar la causa de la autofluorescencia, ya que la celulosa sólo fluoresce generalmente de manera inducida y cuando está mezclada con otros componentes de pared.

Los resultados de este trabajo y otros previos indican, por el momento que los procesos ontogenéticos y la estructura del esporodermo son caracteres relativamente constantes en Equisetum (Beer 1909, Tryon \& Lugardon 1990, Lugardon 1990), algo que implica un valor diagnóstico importante en la taxonomía del grupo (Grauvogel-Stamm \& Lugardon 2009).

El desarrollo de las estructuras esporógenas y esporas de la especie tropical $E$. giganteum, difiere de lo conocido hasta ahora para especies del género procedentes de zonas templadas y podría reflejar una adaptación a diferentes condiciones de humedad estacional, típicas de zonas con escasa amplitud térmica en el año pero con temporadas alternantes de lluvia y sequía. Esta adaptación permitiría la formación y maduración de las esporas durante la estación seca, para ser liberadas masivamente durante el inicio de la temporada húmeda, un proceso que se apoya en caracteres específicos de los equisetos, como la ausencia de dormición, el requerimiento de humedad alta para germinar, la rápida pérdida de la viabilidad y la abundante clorofila de las esporas (Tryon \& Lugardon 1990). Sin embargo, serían necesarios más estudios ecológicos que indagaran sobre los factores externos eventualmente actuantes en la formación y liberación de las esporas de los equisetos tropicales para dar mayor apoyo a esta hipótesis.

\section{AGRADECIMIENTOS}

Agradecemos a la Unidad de Microscopía Electrónica de la Universidad del Cauca (Unicauca), a Dagoberto Arrieta Prieto, coordinador del Laboratorio de Histotécnia de la Universidad Industrial de Santander (UIS) por el préstamo temporal de las instalaciones y al Laboratorio de Morfología Vegetal de la Universidad del Valle (Univalle) por el préstamo de los equipos. A Alba Marina Torres y a Heiber Cárdenas (Univalle) por sus comentarios y sugerencias, y a los revisores anónimos, por las críticas constructivas que contribuyeron a mejorar el manuscrito final.

\section{RESUMEN}

Estudios sobre la ontogenia del estróbilo, los esporangios y la biología reproductiva de Equisetum son escasos, por lo tanto, para la especie E. giganteum, se estudiaron estos aspectos en especímenes recolectados a orillas del Río Frío, Santander, Colombia (2 200m). Los estróbilos en diferentes etapas de maduración fueron fijados, deshidratados, embebidos en parafina, seccionados en micrótomo rotatorio y teñidos con safranina $\mathrm{O}$-fast green. Las observaciones se efectuaron mediante un microscopio óptico de alta resolución con contraste diferencial de interferencia (DIC) y microscopio de fluorescencia. Los estróbilos se inician a partir del meristemo apical, tanto en el eje principal como en los laterales, sin diferencias en el proceso de ontogenia y esporogénesis entre estróbilos de diferentes ejes. Sucesivas mitosis y diferenciación celular conducen al crecimiento del estróbilo, y a la formación de los esporangióforos peltados, formados por el manubrio, o porción basal con aspecto de pedicelo, el escutelo, o porción apical aplanada y las iniciales del esporangio, los cuales se diferenciarán para formar la pared del esporangio, los esporocitos y el tapete. No se forma arquesporio y los esporocitos experimentan meiosis para formar tétradas de esporas. El tapete mantiene la integridad histológica hasta la formación de las tétradas y en esa etapa forma un plasmodio que invade la cavidad esporangial la cual rodea parcialmente las tétradas y luego las esporas, y aparecen las cámaras plasmodiales, un término propuesto aquí para las formaciones designadas en inglés "tapetal gaps". La pared del esporangio queda reducida a dos capas celulares: una externa con engrosamientos lignificados en todas las paredes celulares y una interna picnótica. Al finalizar la 
esporogénesis, el tapete degenera, y las esporas, con exosporio, perisporio delgado, casi membranáceo y eláteres quedan libres en la cavidad esporangial. El esporodermo, los núcleos y nucléolos presentan fluorescencia roja, inducida por coloración con safranina $\mathrm{O}$, mientras que los eláteres y las células de la pared del esporangio presentan autofluorescencia amarillo-naranja.

Palabras clave: Equisetum, estróbilo, ontogenia del esporangio, esporogénesis, esporas, autofluorescencia, fluorescencia inducida.

\section{REFERENCIAS}

Beer, R. 1909. The Development of the spores of Equisetum. New Phytol. 8: 261-266.

Bond, J.L., S. Donaldson, S. Hill \& K. Hitchcock. 2008. Safranine fluorescent stainig of wood cell walls. Biotech. Biotech. Histochem. 83: 161-171.

Currie, H.A. \& C.C. Perry. 2007. Silica in plants: biological, biochemical and chemical studies. Ann. Bot. 100: $1383-1389$.

Currie, H.A. \& C.C. Perry. 2009. Chemical evidence for intrinsic 'Si' within Equisetum cell walls. Phytochemistry 70: 2089-2095.

Chen, C.H. \& J. Lewin. 1969. Silicon as a nutrient element for Equisetum arvense. Can. J. Bot. 47: 125-131.

Des Marais, D.L., A.R. Smith, D.M. Britton \& K.M. Pryer. 2003. Phylogenetic relationships and evolution of extant horsetails, Equisetum, based on chloroplast DNA sequence data $(r b c L$ and $t r n L-F)$. Int. J. Plant. Sci. 164: 737-751.

Duckett, J.G. 1970. Spore size in the genus Equisetum. New Phytol. 69: 333-346.

Epstein, E. 1999. Silicon. Annu. Rev. Plant. Phys. 50: 641-664.

Erdtman, G. 1943. An introduction to pollen analysis. Waltham Massachusetts, EEUU.

Ernst, W.H.O. 1990. Ecophysiology of plants in waterlogged soil. Aquat. Bot. 38: 73-90.

Furness, C.A. \& P.J. Rudall. 2001. The tapetum in basal angiosperms: early diversity. Int. J. Plant. Sci. 162: 375-392.

Furness, C.A. 2008. A review of the distribution of plasmodial and invasive tapeta in eudicots. Int. J. Plant. Sci. 169: 207-223.
Grauvogel-Stamm, L. \& B. Lugardon. 2009. Phylogeny and evolution of the horsetails: Evidence from spore wall ultrastructure. Rev. Palaeobot. Palynol. 156: 116-129.

Guillon, J.M. 2004. Phylogeny of Horsetails (Equisetum) based on the chloroplast rps4 gene and adjacent non coding sequences. Syst. Bot. 29: 251-259.

Guillon, J.M. 2007. Molecular phylogeny of horsetails (Equisetum) including chloroplast atpB sequences. J. Plant. Res. 120: 569-574.

Gullvăg, M. 1968. On the fine structure of the spores of Equisetum fluviatile var. verticillatum studied in the quiescent, germinated and non-viable state. Grana 8: 23-69.

Haseloff, J. 2003. Old botanical techniques for new microscopes. Biotechniques 34: 1174-1182.

Hauke, R.L. 1963. A taxonomical monograph of the genus Equisetum subgenus Hippochaete. Nova Hedwigia 8: 1-23.

Hauke, R.L. 1978. A taxonomic monograph of Equisetum subgenus Equisetum. Nova Hedwigia 30: 385-455.

Hauke, R.L. 1995. Equisetaceae, p. 4-5. In G. Davidse, M. Sousa \& S. Knapp (eds.). Flora Mesoamericana 1. Psilotaceae and Salviniaceae. Instituto de Biología UNAM, Missouri Botanical Garden and the Natural History Museum, Londres.

Hawkins, L.A. 1907. The development of the sporangium of E. hyemale. The Ohio naturalist 7: 122-128.

Holzhuter, G., K. Narayanan \& T. Gerber. 2003. Structure of silica in Equisetum arvense. Anal. Bioanal. Chem. 376: 512-517.

Johansen, D.A. 1940. Plant Microtechnique. McGraw-Hill, Nueva York, EEUU.

Karasawa, R. 1986. Nuclear vacuoles in the sporocytes of five species of musci. J. Hatt. Bot. Lab. 61: 101-108.

Kato, M. 1983. The classification of major groups of pteridophytes. J. Fac. Sci. Univ. Tokyo, Sect. 3, Bot. 13: 263-283.

Kaufman, P.B., D.J. LaCroix \& P. Dayanandan. 1973. Silicification of developing internodes in the perennial scouring rush (Equisetum hyemale var. affine). Dev. Biol. 31: 124-135.

Kenrick, P. \& P.R. Crane. 1997. The origin and early diversification of land plants: a cladistic study. Smithsonian Institution. Washington D.C., EEUU. 
Lehmann, H., K.M. Neidhart \& G. Schlenkermann. 1984. Ultrastructural investigations on sporogenesis in Equisetum fluviatile. Protoplasma 123: 38-47.

Lellinger, D.B. 2002. A modern multiligual glossary for taxonomic pteridology. Pteridologia 3: 1-263.

Lugardon, B. 1978. Isospore and microspore walls of living pteridophytes: identification possibilities with different observation instruments. In 4th Int. Palynol Conf., Lucknow (1976-1977). 1: 152-163.

Lugardon, B. 1990. Pteridophyte sporogenesis: a survey of spore wall ontogeny and fine structure in a polyphyletic plant group, p. 95-120. In S. Blackmore \& R.B. Knox (eds.). Microspores: Evolution and ontogeny. Academic, Londres, Reino Unido.

Ma, Y., V.K. Sawhney \& T.A. Steeves. 1993. Staining of paraffin-embedded plant material in safranine and fast green without prior removal of the paraffin. Can. J. Bot. 71: 996-999.

Majewska-Sawka, A., J. Bohdanowicz, B. Jassem \& M.I. Rodriguez-García. 1990. Development of nuclear vacuolas in sugar beet male meiocytes. Ann. Bot. 66: $139-146$.

Moran, R.C. 1995. Equisetopsida, p. 4. In R. Davidse, M. Sousa \& S. Knapp (eds.). Flora Mesoamericana 1. Psilotaceae and Salviniaceae. Instituto de Biología UNAM, Missouri Botanical Garden and the Natural History Museum, Londres, Reino Unido.

Moran, R.C. 2004. A natural history of ferns. Timber, Portland, Oregon, EEUU.

Murillo, M.T. \& M.A. Harker. 1990. Helechos y plantas afines de Colombia. Academia Colombiana de Ciencias Exactas, Físicas y Naturales, colección Enrique Álvarez Lleras N ${ }^{\circ} 2$ Bogotá.

Olsen, L.T. \& B. Gullvăg. 1973. A fine-structural and cytochemical study of mature and germinating spores of Equisetum arvense. Grana 13: 113-118.

Øllgaard, B. 1975. Studies in Lycopodiaceae, I. Observations on the structure of the sporangium wall. Amer. Fern. J. 65: 19-27.

Pacini, E. \& G.G. Franchi. 1993. Role of the tapetum in pollen and spore dispersal. Plant. Syst. Evol. 7: 1-11.

Pacini, E. 1997. Tapetum character states: analytical keys for tapetum types and activities. Can. J. Bot. 75: 1448-1459.
Page, C.N. 1972. An interpretation of the morphology and evolution of the cone and shoot of Equisetum. Bot. J. Linn. Soc. 65: 359-397.

Parkinson, B.M. 1987. Tapetal organization during sporogenesis in Psilotum nudum. Ann Bot. 60: 353-360.

Parkinson, B.M. \& E. Pacini. 1995. A comparison of tapetal structure and function in pteridophytes and angiosperms. Plant. Syst. Evol. 198: 55-88.

Perkins, S.K., G.A. Peters, T.A. Lumpkin \& H.E. Calvert. 1985. Scanning electron microscopy perine architecture as a taxonomic tool in the genus Azolla Lamarck. Scan. Electron. Microsc. 4: 1719-1734.

Pryer, K.M., A.M. Smith \& J.E. Skog. 1995. Phylogenetic relationship of extant ferns based on evidence from morphology and rbcL sequences. Amer. Fern J. 85: 205-282.

Pryer, K.M., H. Schneider, A.R. Smith, R. Cranfill, P.G. Wolf, J.S. Hunt \& S.D. Sipes. 2001. Horsetails and ferns are a monophyletic group and the closest living relatives to seed plants. Nature 409: 618-622.

Pryer, K.M., E. Schuettpelz, P.G. Wolf, H. Schneider, A.R. Smith \& R. Cranfill. 2004. Phylogeny and evolution of ferns (monilophytes) with a focus on the early leptosporangiate divergences. Am. J. Bot. 91: 1582-1598.

Punt, W., P.P. Hoen, S. Blackmore, S. Nilsson \& A. Le Thomas. 2007. Glossary of pollen and spore terminology. Rev. Palaeobot. Palynol. 143: 1-83.

Renzaglia, K.S., R.J. Duff, D.L. Nickrent \& D.J. Garbary. 2000. Vegetative and reproductive innovations of early land plants; implications for a unified phylogeny. Philos. T. Roy. Soc. B. 355: 769-793.

Rincón, B.E.J., L.V. Gélvez, H.G. Forero, D. Arrieta \& J.S. Hleap. 2009. Ontogenia del esporangio y esporogénesis del licopodio Huperzia brevifolia (Lycopodiaceae) de las altas montañas de Colombia. Rev. Bio.Trop. 57: $1141-1152$.

Rolleri, C. 1972. Morfología comparada de las especies de "Lycopodium" (Pteridophyta Lycopodiaceae) para el noroeste argentino. Rev. Museo La Plata 12: 223-317.

Roshchina, V., V. Yashin \& A. Kononov. 2004. Autofluorescence of developing plant vegetative microsporas studied by confocal microscopy and microspectrofluorimetry. J. Fluoresc. 14: 745-750.

Ruzin, S.E. 1999. Plant microtechnique and microscopy. Oxford University, NuevaYork, EEUU. 
Sheffield, E., A.H. Cawood, P.R. Bell \& H.G. Dickinson. 1979. The development of nuclear vacuoles during meiosis in plant. Planta 146: 597-601.

Sheffield, E., S. Laird \& P.R. Bell. 1983. Ultrastructural aspects of sporogenesis in the apogamous fern Dryopteris borreri. J. Cell. Sci. 63: 125-134.

Schuettpelz, E., P. Korall \& K.M. Pryer. 2006. Plastid atpA data provide improved support for deep relationships among ferns. Taxon 55: 897-906.

Schuettpelz, E. \& K.M. Pryer. 2008. Fern phylogeny, p. 395-416. In T.A. Ranker \& C.H. Haufler (eds.). Biology and evolution of ferns and lycophytes. Cambridge University, Cambridge, Reino Unido.

Smith, A.R., K.M. Pryer, E. Schuettpelz, P. Korall, H. Schneider \& P.G. Wolf. 2006. A classification for extant ferns. Taxon 55: 705-731.

Triana-Moreno, L.A. \& J.A. Murillo. 2005. Helechos y plantas afines de Alban (Cundinamarca): El bosque subandino y su diversidad. Instituto de
Ciencias Naturales-Universidad Nacional de Colombia, Instituto de Investigación de Recursos Biológicos “Alexander von Humboldt”. Bogotá D.C. Colombia.

Tryon, R.M. \& A.F. Tryon. 1982. Ferns and allied plants with special reference to tropical America. Springer, Nueva York, EEUU.

Tryon, A.F. \& B. Lugardon. 1990. Spores of the Pteridophyta: Surface, wall structure, and diversity based on electron microscope studies. Springer, Nueva York, EEUU.

Uehara, K. \& S. Kurita. 1989. An ultrastructural study of spore wall morphogenesis in Equisetum arvense. Am. J. Bot. 76: 939-951.

Uehara, K. \& S. Kurita. 1991. Ultrastructural study on spore wall morphogenesis in Lycopodium clavatum (Lycopodiaceae). Am. J. Bot. 78: 24-36.

Uehara, K. \& S. Murakami. 1995. Arrangement of microtubules during spore formation in Equisetum arvense (Equisetaceae). Am. J. Bot. 82: 75-80. 